\title{
Exploring the benefits and challenges of administrative clinical supervision in nursing and midwifery
}

\author{
Gloria Achempim-Ansong ${ }^{1}$, Atswei Adzo Kwashie ${ }^{* 2}$, Adelaide Ansah Ofei ${ }^{2}$ \\ ${ }^{1}$ Department of Adult Health Nursing, School of Nursing and Midwifery, University of Ghana, Ghana \\ ${ }^{2}$ Department of Research, Education and Administration, School of Nursing and Midwifery, University of Ghana, Ghana
}

Received: June 29, 2021

DOI: $10.5430 /$ jnep.v12n1p14
Accepted: August 16, 2021

Online Published: August 27, 2021

\begin{abstract}
Objective: To explore the benefits and challenges of administrative clinical supervision at the unit.

Methods: Clinical Supervision has been recognised as a core component of professional support for present-day nursing and midwifery practice. Effective administrative clinical supervision is beneficial to employees by providing support leading to the development of self-esteem, reducing emotional stress and improving commitment to an organisation's vision and goals. Using aesthetic phenomenological approach, nurses' lived experience with administrative clinical supervision in a clinical unit of the hospital was explored and allowed in-depth description of administrative clinical supervision thoroughly. The participants were purposively sampled from six hospitals in Accra and comprised supervisors $(n=18)$ and supervisees $(n=12)$.

Results: The benefits of administrative clinical supervision include reduction in infection rates, improved competence, client satisfaction, reduction in negligence, efficiency, accountability and feeling of being appreciated. Challenges of administrative clinical supervision were managerial challenges, limited time, interpersonal conflict with colleagues, and increased workload.

Conclusions: Practical Implications: Administrative clinical supervision has implications for nursing and midwifery education and practice. This calls for measures that promote practitioners' personal and professional development through fostering a supportive relationship and working alliance. Originality: This study employed the use of Aesthetic Phenomenology to tell the stories of clinical supervision from the personified interpretations unlike other studies that simply adopt descriptive phenomenology. It is the first of its kind in Ghana to the best of our knowledge.
\end{abstract}

Key Words: Administrative clinical supervision, Benefits, Challenges, Aesthetic phenomenology

\section{INTRODUCTION}

Clinical supervision (CS) involves a supportive relationship between supervisor and supervisee that facilitates reflective learning and is part of professional socialisation. ${ }^{[1]}$ It can take various forms and requires that the supervisor and supervisee agree to work in an environment that fosters learning leading to job satisfaction and less stress. Unlike clinical supervision of student nurses to acquire requisite knowledge and skills for future practice, the administrative aspect of CS focuses on a formal supportive professional development process contributing to the personal growth of a practicing nurse. ${ }^{[2]}$ For the purposes of this paper, CS refers to administrative clinical supervision. The complexity of the clinical supervision process is such that it requires the administrative head of a unit to be skilled in CS to ensure patient safety and to ensure competency of the supervisee. The tutelage provided

\footnotetext{
*Correspondence: Atswei Adzo Kwashie; Email: aakwashie@ug.edu.gh; Address: Department of Research, Education and Administration, School of Nursing and Midwifery, University of Ghana, Ghana.
} 
in supervision can come from three separate but interconnected forms of supervision: educational, administrative, and clinical. ${ }^{[3]}$ Administrative CS include the management of staffing and productivity, assessing supervisee and patient needs, completing quality assurance and compliance reviews among other tasks within the clinical setting to improve clinical practice. Thus, CS has been described as involving a conversation which facilitates reflective learning through a non-judgemental process. ${ }^{[4]}$ Thus, CS is necessary for effective practice to enable delivery of quality care in healthcare settings. In nursing and midwifery, CS is done by active engagement between supervisor and supervisee in a relationship that fosters confidence and trust. Often the supervisor is a senior nurse practitioner with the supervisee being a newly qualified nurse or one with few years of practice.

CS has been associated with higher levels of job satisfaction, improved retention, reduced turnover and staff effectiveness. ${ }^{[5]}$ Furthermore, it reduces emotional stress, enables reflective practice as well as providing support and encouragement. These lead to the development of self-esteem and self-confidence in the supervisee. Effective clinical supervision may increase employees' perceptions of organisational support and improve their commitment to an organisation's vision and goals. It also enhances staff retention and improves workplace morale. Importantly, CS has been linked to effective clinical governance, by helping to support quality improvement, managing risks, and by increasing accountability. ${ }^{[3]}$ These benefits of CS facilitate the development of nursing leadership and the opportunity to critique clinical and cultural practices within a safe environment. ${ }^{[6]}$ Good CS relies on trust and therefore a supervisee has a right to expect the content of the session to remain confidential.

Driscoll et al. ${ }^{[4]}$ recounted several benefits of CS for staff as helping staff to manage the personal and professional demands created by the nature of their work, providing an environment for exploring personal and emotional reactions to work, allowing staff to reflect on and to challenge their own practice in a safe and confidential environment and to also receive feedback on their skills that is separate from managerial considerations. CS can be part of professional development, and a way to identify developmental needs. This regular process of support and development of the supervisee within the safe space of the clinical setting improves care delivery while offering support to the supervisee to be used in reflective practice and revalidation. Although, there are many benefits that can be reaped from effective CS, limited resources, lack of knowledge or competence on the part of those who are supposed to be supervisors, ineffective communication, etc., are some documented stumbling blocks to effective CS. Again, the lack of practical implementation of problems, lack of management support and the low level of availability of CS for nurses working under high-risk conditions also tend to negatively affect effective administrative CS programmes. ${ }^{[4,7]}$

Barriers to effective implementation of CS are personal and organizational. Ning and Costello ${ }^{[2]}$ indicated that personally, it includes a fear of change and a lack of confidence, knowledge skills or understanding, resulting in the lack of a common understanding of the nature or purpose of CS and ad hoc implementation models. Organizational issues include lack of resources, funding or time and issues of management support. ${ }^{[8]}$ It was established that through empowerment and fair leadership CS in nursing enhances self-confidence, commitment, and competence. Nursing has a strong culture of professional support but the immediate access to informal support may hinder the uptake of formal CS. ${ }^{[9]}$ Problem solving in line with Proctor's restorative function that takes place via informal meetings usually goes unrecognized. ${ }^{[10]}$

As a professional development activity, CS ensures that less experienced clinicians use the knowledge and experience of supervisors to dovetail any gaps in knowledge or skill set to develop their clinical proficiency. However, several approaches can be adopted for CS to suit the local circumstances, or a working agreement can be established between the parties. According to Lyth, ${ }^{[1]}$ many practitioners remain unsure about the concept of CS and what is expected of them. Tomlinson ${ }^{[12]}$ also argued that if CS is to be recommended, the packaging should be clarified whereas, several researchers have attempted to conceptualize CS as senior clinicians overseeing and guiding the practice of less experience clinicians. ${ }^{[13-15]}$ The study thus, explored the benefits and challenges of administrative CS of supervisors who are usually ward managers (ward in-charges) in the unit.

\section{Methods}

\subsection{Design and participants}

An aesthetic phenomenological approach ${ }^{[16]}$ was used to explore nurses' lived experience to provide an exhaustive description of their concerns regarding the concept of CS at the unit level. Using phenomenological inquiry, projected aesthetic comprehension of the phenomenon of CS at the unit level was accomplished. Aesthetically, nurses' experience is explored using their own words, the expression of their opinions (communicative self-reflection), the import attached to their daily engagement with supervisors or supervisees in the unit, and their sentiments regarding imbibing the humanistic culture of nursing. The entire process intrinsically prevailed by the researchers' instincts of probing for in-depth information about the phenomenon of CS. This design allowed us to capture the complexity and richness of the lived experiences 
and concerns of nurses.

Individual in-depth interviews with supervisors $(\mathrm{n}=18)$ and supervisees $(n=12)$ took place in six hospitals in Accra. Participants were all nurses working in the unit. Inclusion criteria included; (1) professional nurses, (2) should have worked for at least one year, (3) should have worked in the hospital for at least six months.

\subsection{Setting and data collection}

Data was collected from six selected hospitals in Accra: one teaching hospital, one specialized institution, one secondary facility, and three primary facilities, depicting the levels of healthcare delivery in Ghana. Participants were identified and selected from the units throughout all the shifts. The research team explained the intent of the study, the possibility to withdraw from the study if they so wished, with no repercussions as well as the roles and responsibilities of participants. Ethical issues regarding research with human participants were explained and ensured by allowing participants to ask questions about the study, to allay anxiety and signing written consents. Participants' confidentiality was protected throughout the research process by using pseudo names to protect identity and privacy of participants. The in-depth face-to-face interviews allowed the participants to freely discuss their experiences and concerns and each interview lasted for about 60 minutes.

Institutional permission was used to purposively select nurses (supervisors and supervisees) from the hospitals. The supervisors were nurse managers with 10 to 20 years of work experience whereas, the supervisees were nurses with 1 to 10 years of work experience in the unit. The interviews were digitally recorded and transcribed verbatim and participants were given the opportunity to member-check their transcription to ensure it matched their memory of the interview.

\subsection{Data analysis}

Concurrent manual analysis of data was used to manage the volume of data and to facilitate the accessibility of raw data for content analysis and personified interpretation. The process of deciphering the aesthetic data into findings is driven by an epistemological framework grounded in the philosophy of content analysis. Participants' own phrases and words were used within the personified interpretation to ensure that the findings reflected the exact experiences of the participants. The researchers repeatedly listened to the recordings, read, and reread the transcripts, as well as the immersion of the researchers in the words used by the participants, in their meanings and expressions to select phrases that capture the combined and similar experiences and meanings of the participants.
Using personified interpretation and adhering to the epistemological framework, themes and subthemes within the perception of supervision transcripts were identified from the experiences and concerns of supervisors and supervisees. These themes and subthemes subsequently provided insight into participants' concerns about supervision in the unit, and an aesthetic descriptive interpretation was espoused to personify the findings in a way that arouses a collective comprehension of their meaning. Thus, intensely pursued by persistent reading between words and their felt complexity in the lived experiences of participants, the commonality between the participants' experiences and concerns were presented using their individual thoughts from the data. The data captured the participants' perception and meaning thus, the information was not transformed but remained true exhibits of the original intentions of supervisors' and supervisees' shared experiences and thoughts.

\subsection{Trustworthiness}

Trustworthiness was ensured by the development and refinement of the interview guide through a pilot study. Ethical clearance was obtained from the School of Nursing and Midwifery, University of Ghana (SONM/REA/01-2019). The subjectivities, biases and particularities of researchers were moderated by enabling accuracy (validity) and credibility (reliability) of the findings. The reliability of the study was ensured by audio trail; interviews were played back to participants for reflection and for audition of their shared experiences and concerns. Individual transcripts were also reviewed consistently by the researchers to ensure the exact data has been captured. The researchers ensured there was no drift in the definition of codes or a shift in the meaning of the codes by constantly comparing data and by keeping account of the codes and their meaning; that is consistent cross-checking to ensure consistency of the coding.

Validity strategies were actively incorporated to ensure accuracy of findings using triangulation, member checking, external auditor, and spending of prolonged time in the field. ${ }^{[1]}$ Data from the different hospitals, supervisors and supervisees was triangulated to develop a coherent justification for the themes and subthemes. Converging the two dimensions of data from both supervisors and supervisees added validity to the study. Member checking was done by availing the final report of themes and subthemes to participants for validation of the accuracy of the narrative of their lived experiences and concerns. Colleagues of the researchers were used as external auditors to review the entire process, objectives, review of literature, and methods especially, data analysis to strengthen the consistency of the study. Again, the researchers spent a long time (four months) in the field collecting data from 
thirty participants from six hospitals. This provided the researchers with an in-depth comprehension of CS thus, the reason for interviewing both supervisors and supervisees. This projected a comprehensive description of lived experiences and concerns of participants about CS in the unit. A wider view of reflexivity is evident in epistemological reflexivity where the researchers interviewed participants based on questions aligned with their methodological decision making regarding the research and its findings.

\section{Findings}

\subsection{Demographic characteristics of participants}

Thirty participants were interviewed for the study, 18 supervisors and 12 supervisees. The supervisors' designation ranged from Deputy Director of Nursing Service (1), Principal Nursing Officers (7) to Senior Nursing Officers (10) whereas, the supervisees were Nursing Officers (7), Senior Staff Nurses (3), and Staff Nurses (2). Age for the supervisors ranged between 46 and 32 years while the supervisees' age ranged between 24 to 35 years. There were two (2) males and twenty-eight (28) females. The supervisors and supervisees both have a variety of clinical practice backgrounds ranging from midwifery, general nursing, psychiatry, emergency to paediatric nursing.

\subsection{Benefits of supervision}

Supervision is very prominent in nursing practice and facilitates the transfer of knowledge, skills, and attitudes among professionals. Immersion into the theme on benefits of supervision had several subthemes emerging from the data. The subthemes are reduction in infection rate, reduction in negligence, improved competence, good corporate image, efficiency, accountability, client satisfaction, feeling of being appreciated, learning to deal with diversity and being familiar with the environment.

Participants believed that with good supervision, there could be reduction in infection rate. This is a clear indication that proper supervision in nursing and midwifery practice contributes to positive patient outcomes such as reduction in infection rate.

"Like, reduction in infection rate." (NSB2)

Participants also perceived that supervision promotes reduction in negligence as supervisees would do the right thing since the supervisor would be vigilant in monitoring the work of supervisees. A vigilant clinical supervisor ensures that practices of the staff meet the required standards to avoid mistakes.

$$
\text { “... reduction in nursing negligence, medica- }
$$$$
\text { tion errors and all that. I think that if you don't }
$$

have a good supervisor, you will hear more of such things. That is something that is really significant in our profession because she keeps an eagle eye on what is being done."(NSB2)

Some participants were of the view that good supervision leads to effective learning which improves competence and skilfulness of supervisees. This also improves care leading to client satisfaction as acknowledged;

“... good supervision would make them(nurses) learn a lot of things, to become very competent, skilful, and having our patients very satisfied with our care.'(REA1)

Good corporate image was another subtheme that emerged from the data. Good supervision increases the number of clients that utilize the hospital due to the provision of quality services by staff. This increases the revenue generated by the hospital and raises the level of the hospital, that is, improvement of the hospital's corporate image. REA2 stated that:
"One, the hospital will have a name, there would be a lot of people visiting the hospital. Again, the infrastructure will be improved, the financial status of the hospital would be raised and then the level of the hospital will also be raised."

Another subtheme that emerged from the theme benefits of supervision was efficiency and satisfaction with care provided to patients. Effective clinical supervision ensures that good work is done and at the end of the day everyone is satisfied; the nurse is satisfied, the patient is satisfied. This improves the working standards thus, work progresses well with positive outcomes. Some comments generated from some participants included:

“... with effective supervision good work is done and at the end of the day everybody goes away satisfied. The nurse becomes satisfied, the patient is satisfied with the care because the work is being done."( REA1)

"One is efficiency and then it improves the working standards because if the work is supervised very well, everything is done well."( REA2)

"Work progresses well, there is good outcome. Because we are talking about patients, patients care outcomes are good. Without supervision you realize that patients stay longer on the ward, they are not cared for properly, they are not health educated and that has more lasting effects. But once there is supervision, and an effective 
one, even if you have few supplies they are distributed appropriately, and they are taken care of properly"( REA3)

Good supervision also ensured accountability among staff since the supervisor would demand feedback for work done from supervisees. Client satisfaction with care was of paramount importance to the participants and they expressed it through the numerous quotes extracted from the data. Participants believed that with good supervision, clients become satisfied with care rendered.

Another sub-theme from the data was that of 'feeling appreciated'. Clinical supervisors expressed a sense of feeling appreciated by the supervisees during CS. As a supervisor, the individual benefits not just from salary increment or incentives (though it's important), but there is the feeling of being appreciated by supervisees, as well as the feeling of people coming to you to seek for solutions to problems. A clinical supervisor recounted that:

"I would say yes, I have gained some benefits as a supervisor not just salary increment or incentives even though it's part, the feeling of being appreciated, the feeling of the people coming to you to seek for solutions or demand for certain things that need to be done. They put you at the forefront to do things for them. It gives you great pleasure, and it makes you want to do more so that you can always help people. So, I think being a supervisor has given me that pleasure.’(CIND3)

Another key benefit that emerged was learning to deal with diversity. Supervising people is a learning process that deals with different kinds of characters and people. By engaging in the process of CS, you find ways of dealing with such diverse people. This grooms you for other opportunities.

"I think once people are placed under you to supervise, it's a learning process. You deal with different kinds of characters and people, so you find ways of dealing with such people. So, actually it grooms you as a person for other opportunities."(CIND2)

The final subtheme to related to benefits of CS was 'becoming familiar with the environment'. Participants believed that because the supervisor goes around to supervise, he/she becomes familiar with the environment and gets to intervene to make things right. Clinical supervision requires that the supervisor moves around the facility from one unit to the other. This provides the opportunity to become very familiar with the environment in which one works. That way, the supervisor gets to know what is happening at the unit instead of receiving reports from third parties. Knowing about problems first-hand allows for the right interventions to be put in place for prompt intervention. This was expressed in the quote;

"Yes, because when you go on supervision you see what is going on the ground and then you come in or you intervene to make things go right. Also, for myself I also see where I need to buck up and then I put in those measures."(CIND1)

\subsection{Challenges of supervision}

The challenges of clinical supervision were expressed mostly by the clinical supervisors themselves. They presented challenges they faced during the process of clinical supervision. Numerous subthemes emerged under challenges of supervision. These are, no right to lead, miscommunication, limited time, interpersonal conflict with colleagues, increased workload, fatigue, inadequate staff, lack of interest among supervisees, and lack of trust. The remaining subthemes are inadequate logistics, unconstructive criticisms, managerial challenges, administrative challenges, learning and development, inadequate equipment, stubborn subordinates, sabotage, unwilling to accept criticisms, ineffective communication, people not doing the right thing, and backward culture.

Participants believed that there are lots of challenges that confront the leader at the workplace especially, if the leader is young. A young clinical supervisor is seen as having "no right to lead" supervisee who may be older than him/her. For instance, CIND3 asserted that:

"Some people feel I (supervisor) have no right to be their leader because probably, I am younger than them."

Another challenge that confronts the administrative clinical supervision process is limited time. Participants acknowledged that once in a while, limited time prevented them from supervising subordinates because, as supervisors, they have other things to do and may not pay attention to subordinates. Additionally, when confronted with a 'difficult staff' who may refuse to comply with instructions, supervisors tend not to spend the limited time on such persons. In addition, supervision is time-consuming, and one needs enough time to ensure that everything is done well. Comments from participants included:

"Once in a while, there is limited time on my part, maybe I may have to do other things in the 
office. When the time is limited, I am not able to go on supervision and then meeting a difficult staff who is refusing to comply with what you are saying. That is also a little challenge." CIND1

"So, when they are supervising, they don't have the time, and do not pay attention to their subordinates." TATA3

"And you know it's time consuming being a supervisor. You need to be on time, make sure that everything is done. You have to be sure that, whatever objectives are set have been met." LH3

Interpersonal conflict with colleagues is another challenge that emerged because colleagues don't accept that once they are all professionals there is a need for one person to lead, give instructions or supervise to ensure activities are accomplished accordingly as per the hierarchy in the nursing profession. A supervisor had this to say:

"Yes, yes, sometimes your own colleagues would think, "why should my own colleague instruct me, being a midwife just like herself. But we have hierarchy in the nursing profession so when we come on duty we have a shift-incharge though we are all colleagues. In times of any trouble, management would come for the shift-in-charge first to answer. So, when you instruct somebody to do this or that, they think you are being bossy and all that thinking to themselves, "I can do it, why is she instructing me?". But that is not the case. We need to save lives and all that. If you(subordinate) are not doing something right, I'll instruct you in my capacity as the shift-in-charge. That way, we all have a peaceful day, a peaceful shift, and can all go home peacefully while saving more lives at work."(AYD3)

Increased workload was one challenge that also emerged from the data. Participants complained about inability to supervise due to increased workload especially, documentation, which ushers in fatigue and inability to accomplish tasks well. Participants said that sometimes there is so much work to be done by the few staff available on duty. In this case, the supervisor, tend to avoid supervision and rather focus on ensuring that the work done. Some participants had these to say:

"... most of the time the supervisors have a lot of work to do and also a lot of things to clear on their desk. They are unable to supervise us." TATA3

"The workload is heavy; you have to do documentation; you have to make sure that all the clients' particulars are set. For the midwives also, sometimes the workload coupled with and the number of clients to work on is heavy. They focus more on attending to clients rather than teaching, as teaching will waste time. They have to see to every client who is in labour or needs care, thus, they find teaching a bit difficult." AYD2

“... sometimes when there is so much work to be done by few staff, you'll decide to work rather than engage in clinical supervision. You can't control the ward with only this few number of staff. So, I think that gives me reason to avoid clinical supervision at that point in time." CIND3

"Most of the time the supervisors have a lot of work to do and a lot of things to clear on their desk." TATA2

Some participants acknowledged that fatigue is a challenge that confronts supervision. This results in low productivity and poor patient outcomes. The workload may be quite high to the extent of supervisors not being able to cope. Often, they break down and ultimately experience burnout. A participant asserted that:

"The challenges are numerous, and you get burnout and fatigue because you want productivity and good result. At the end of the day, you even breakdown especially, at the clinical area, it's so challenging." (AP3)

Participants also agreed that inadequate staffing is another challenge for the supervision process. Units were understaffed in terms of professional nurses. Rather, there are a lot of health assistants working in the unit instead of registered nurses. With many patients to provide care for, teaching of subordinates becomes more difficult. Participants stated that:

"When it comes to the number of staff working here, we are understaffed. The qualified personnel as in the diploma nurses are not available. We have a lot of the health assistants but the qualified nurses, we don't have enough of us working here. And the ward is too big. It is huge. And the work here is so much. We need to get it done too so if there's a little oversight, trouble for you." DBD1 
"The staff strength is not enough, and the clients are more than the staff that is where teaching becomes a bit more difficult because we'll not like to waste time on client teaching." AYD2

"Yes, also the human resource is limited, sometimes some have to go on leave, maternity leave etc.” LH1

"Well, sometimes you are not able to supervise effectively because we are not many." REA1

Lack of interest among supervisees is another subtheme that emerged from the data. Participants acknowledged that supervisees are only interested in the outcome of a procedure but not to take time to learn about the process. Participants also acknowledged that supervisees who are not ready to learn pose problems when it comes to supervision. Either they are in the profession for a different reason or just not passionate about the work. It becomes very stressful when dealing with such people because they keep on making mistakes and committing minor errors that the supervisor will have to deal with.

"And then with the supervisee, the only thing on their mind is to see a delivery; "I want to see a baby coming". It's a little bit challenging to sit them down to teach them. They are not ready to sit down to learn.”(AYD2)

"Sometimes when you are dealing with supervisees who are not ready to learn, who are just unwilling, no matter what you do they are just so demotivated and there is nothing you can do about them. Either they are in the profession for a different reason, or they just don't have the passion for the work. It is very stressful when you are dealing with such people because they keep on making mistakes and committing minor errors you always have to deal with." (NSB2)

Lack of trust as a subtheme also emerged. The participants were of the view that if the task is a challenging one for the supervisee, it would be difficult to trust that the individual will come out with the needed results. This is what a participant had to say:

"If you realize that the task ahead is a challenging one for the supervisee it will be difficult to trust that once you ask them to go ahead with it, you will actually get the needed results. So, that has been one of the few challenges that I have encountered. Supervisees who are not necessarily in the best of shapes to carry out an assignment."(AYD1)
Inadequate logistics is another challenge of CS. Items needed in the unit to perform tasks are normally unavailable, making work ineffective. Supervisors had to struggle to search for adequate resources for work in the unit. Participants had this to say:

“... sometimes the necessary items, the necessary things needed to perform tasks are not there so, it makes it very difficult to perform the task. Sometimes because of that, you are not able to do it effectively or as you ought to do." VA3

“..., on few occasions, one faces logistical challenges. Subordinates do not have the needed logistics to actually carry out their duties." AYD1

"She (the supervisor) has to make sure that we have logistics available for us to work with. ... when it comes to adequate resources, thus, she must make sure that she gets resources for us to perform our duties. Most of the time the logistics are not there. She is responsible for handling the staff on the ward." DEDE1

Criticism of work done serves to correct mistakes and enhance one's performance of duties that are assigned to the individual. When done in a constructive manner, it fosters learning which brings about competency in clinical skills. Participants mentioned that unconstructive criticism is one challenge that confronts CS. Supervisors sometimes do not criticize constructively as expressed in the quote by a participant.

"Sometimes too, they( the supervisors) don't criticize constructively”.(TATA2)

Learning and development as a subtheme looks at the difficulty in organizing training for staff due to the shift system. Clinical supervision involves provision of opportunities for learning and personal development of the supervisees. Training programmes are organised to update staff on new developments and trends in clinical practice. However, this was a challenge for the supervisors due to the nature of their shifts which makes it difficult to get the right participants to be part of the programmes. This was confirmed in the quote:

"Sometimes it is not easy organizing staff to get them trained due to the shift work that we do. All the same, we sometimes get a few people around and we use them to spread the knowledge acquired from the training." (TATA3)

Support system (maintenance) as a subtheme refers to the outcome of the unit's inability to 
assemble some equipment to support on-going work especially by way of maintenance of equipment. This was expressed by some participants who stated that: "The support system in the hospital is also another challenge, especially in situations where you really need certain equipment and things to support your work and they are not forthcoming." (TATA1)

“... availability of some of the equipment are also a challenge. We need to have them and also know how to fix them. At times, when you call the maintenance team, they would say they are coming. They won't come promptly. Meanwhile this place(unit) is an emergency, so we need to be ready for all eventualities." REA2

The subtheme 'stubborn subordinates' refers to description of the behaviour and attitude of some subordinates, and the fact that these subordinates do not adhere to rules, or do not complete their assignment which leads to a lot of pressure and lack of trust at work. Supervisors sometimes have to deal with such DEDE2 affirmed that:

"Stubborn subordinates who do not adhere to rules that are laid down. Subordinates do not complete their assignments and there is a lot of pressure. If you don't get good and trusting subordinates, it brings about a lot of workload and pressure on the supervisor."

Sabotage of the clinical supervisor's work is another subtheme that emerged from the data. Participants acknowledged that if the supervisor does not meet the learning needs or interests of supervisees, they try to sabotage the supervisor's work making the individual ineffective. A participant had this to say:

"If you do not try to meet the interests of your supervisees, then they will try to sabotage your work. At the end of the day, it will not make you an effective supervisor." (DEDE1)

Participants also accepted that sometimes supervisees do not have the ability to accept criticisms for change of approach towards work. Supervisees become emotional and take offence at being corrected which makes supervision quite challenging. Participants acknowledged that:

"Sometimes too the emotions, because not everyone has the ability to accept criticism and make a change. So sometimes when you are dealing with very emotional people who take offence at correction, it can also be a challenge for supervision." (NSB2)

"Sometimes the attitudes of the individuals, some you know in the course of supervision, you may want to correct in a nice way, but some don't have the disposition to be corrected." (LH1)

The old ways of doing things in the nursing and midwifery profession leads to stagnation which participants expressed as 'backward culture'. Healthcare is dynamic and keeps evolving. However some professionals are unable to adapt to these changes. Participants believed that even though the nursing profession is evolving, some nurses still want to maintain the old ways of doing things. They therefore saw this 'backward culture' as a challenge with clinical supervision. LH2 confided that:

“... nursing is evolving but people still have the attitude of Florence nightingale, (in Twi) this is how it is done and that is it. So, if you try to bring a change, you become a victim of circumstances. So, if I also don't want to have problems, if you don't take time you might go like the way they want, you to go and it doesn't help."

\section{Discussion}

In this phenomenological inquiry, we explored the lived experiences of nurses regarding the concept of clinical supervision at the unit level. Nurses' experience is explored using their own words, the expression of their opinions, the import attached to their daily engagement with supervisors or supervisees in the unit, to provide an exhaustive description of their concerns and sentiments regarding imbibing the humanistic culture of nursing. This study enabled the identification of some benefits and challenges of clinical supervision and these findings can be compared with other research. The literature identifies a whole range of benefits and challenges of CS.

The study identified that CS promotes reduction in infection rate. Infection prevention and control measures are instilled in supervisees and supervisors ensure that these measures are strictly applied appropriately. Over time and through constant practice, the supervisees graduate from novice to experts in infection prevention thereby, reducing infection rate and promoting safety at the clinical unit. For instance, Martin et al. ${ }^{[7]}$ acknowledged that patient outcomes are improved and infection rate reduced when health professionals are clinically supervised. It has also been observed that CS benefits patient outcomes ${ }^{[18]}$ by having a positive effect on 
patient outcomes which include infection prevention. According to Martin et al. ${ }^{[18]}$ lack of clinical supervision of health professionals can be fatal for the patient if infection prevention is not ensured.

Interviewees in our study also highlighted that CS promotes effective learning. Supervisees become more focussed and eager to learn with proper supervision resulting in improved competence, more knowledgeable and skilful. Supervisees also learn to adopt some positive attitudes portrayed by supervisors. Similarly, King et al. ${ }^{[19]}$ also found skill acquisition as one benefit of CS in their study. They however averted that to promote effective learning and skill acquisition by supervisees, there is the need for a conducive environment at the clinical care unit.

Reduction in negligence was also identified as a key benefit of CS. Supervisees ensure the right thing is done since the supervisor keeps an eagle eye on whatever is going on to ensure that procedures are done correctly to prevent chaos. The reduction in negligence mentioned by interviewees in our study is also echoed in the literature. For example, Ekanoye ${ }^{[20]}$ also revealed in a qualitative action-based research among supervisees in Nursing and Allied Health in Australia that CS reduces negligence thereby, promoting safety at the clinical unit.

Good corporate image was also intimated by participants as an important benefit of effective supervision. Clinical supervision when done effectively helps to maintain the reputation of the facility and gives a better picture of the image that people hold in their mind about the facility hence, services provided will influence their patronage of the facility. With good CS, supervisors ensure that supervisees work according to the standard protocols, thereby, enhancing the facility's image and promoting patients' safety in the unit. Several studies have asserted good corporate image as a significant benefit of clinical supervision. ${ }^{[21,22]}$ Quality control, creativity, and professionalism are depicted, making the facility attractive to consumers.

Participants also believed that with good supervision, accountability among staff is ensured. Supervisees become more responsible since they know that they are accountable for all their actions since supervisors demand feedback for work accomplished. Strong corporate governance practices foster a workplace culture built on high standards of accountability, responsibility, and integrity. The findings of our study support previous research by Snowdon ${ }^{[23]}$ which also revealed that CS improves adherence to acceptability and repeatability criteria for performance of certain procedures and promotes accountability in the clinical setting.
Again, participants acknowledged that CS breeds efficiency in the unit. They believed that with effective supervision, good work is done as supervisees accomplish the right task in the right way with least time and effort resulting in nurse and patient satisfaction. This improves the working standards, for there is better supervision, hence tasks are performed well. Thus, the work in the unit progresses with good outcomes. Similar benefits of CS are well documented by literatures. ${ }^{[23-25]}$ For instance, a qualitative study reported that CS enables nurses and midwives to practise effectively in a complex health system, enhancing the quality of care. ${ }^{[26]}$

We also recognised that with good supervision, supervisors learn to deal with diversity as they manage diverse individuals in terms of gender, race, ethnicity, age, sexuality, language and educational background. It is a social responsibility for supervisors to embrace diversity at the workplace since it ushers in better prospects and productivity, making supervisees' expereinces fulfilling. Supervising people is a learning process that deals with different kinds of characters and people, so the supervisor develops ways of dealing with such people. Novoa-Gómez et al. ${ }^{[27]}$ also revealed in a similar study that supervisors deal with supervisees from different backgrounds, hence, learn to embrace diversity. They however opined that; skills related to the clinical supervisors' duties should be extensively conceptualized.

Consistent with previous findings Ziba et al. ${ }^{[28]}$ also emphasized familiarity with the clinical environment as one benefit of CS. They believed that the supervisor becomes familiar with the environment, sees what is happening and gets to intervene to make things right during supervision.

While CS was perceived as having several benefits, various challenges were iterated by participants. These primarily were miscommunication and ineffective communication, limited time, increased workload, fatigue, inadequate staff and logistics, lack of trust and interest among supervisees, unconstructive criticisms, managerial and administrative challenges, interpersonal conflict with colleagues, stubborn subordinates, sabotage, unwillingness to accept criticisms, and backward culture. In the Ghanaian context, these challenges often arise because culturally, higher rank is associated with age. However, advanced academic qualification may ensue in a situation where younger persons assume the position of clinical supervisors. Hence when older nurses or midwives must be supervised by such supervisors, the plethora of challenges that emerged from the data are not uncommon. Some literature are consistent with these findings particularly in contexts that have similar cultural backgrounds. ${ }^{[28-30]}$

Participants alluded that there is always some form of miscommunication when individuals are asked to perform tasks 
and they can't really comprehend what they have been asked to do. Ineffective communication was also mentioned by participants as a challenge. Supervisees sometimes do not see the 'big picture' and are only told what they are supposed to do. These findings align with those of Ekanoye ${ }^{[20]}$ who also revealed that ineffective communication as well as language barrier hinder effective CS.

Like other research findings ${ }^{[26,32,33]}$ participants in this study revealed that inadequate staffing, time constraint and increased workload pose as barriers to effective CS. Participants stated that increased workload especially, cumbersome documentation processes coupled with understaffing of units inhibit CS since clinical facilitators experience fatigue and burnout, hence, tend to avoid supervision.

Lack of logistics was also underscored by participants as a challenge of CS. Items needed to perform tasks are often not available. Nurses have to resort to improvising. Lack of logistics was also echoed in the literatures. ${ }^{[26,32]}$ There should, therefore, be adequate provision of resources to facilitate performance. Participants also believed that backward culture was a barrier to effective CS. They believed that even though nursing profession is evolving, some nurses still want to maintain the old ways of doing things. In line with this finding, Saab et al. ${ }^{[26]}$ revealed that lack of buy-in as well as the culture of maintaining old ways of doing things were some challenges of CS. Ekanoye ${ }^{[20]}$ also identified acculturation as a challenge to effective CS. The fear of trying new things often inhibit the use of new ideas, it is however, that we incorporate evidence of good practices into our work to enhance patient satisfaction.

Lack of trust and interest among supervisees, unconstructive criticisms, managerial and administrative challenges, interpersonal conflict with colleagues, stubborn subordinates, sabotage, unwillingness to accept criticisms were also emphasised by participants of the study as some challenges of CS. Some subordinates do not adhere to laid down rules, complete assignments and do not also accept criticisms since supervisors often do not criticise constructively. These factors may pose as challenges to clinical supervision. In accordance with these findings, Long et al. ${ }^{[9]}$ also alluded that personal and organisational factors constitute a challenge to CS. In support of the present study, Tomlinson, ${ }^{[12]}$ also purported that lack of appropriate values, attitudes, and behaviours on the part of both supervisors and supervisees may render CS ineffective.

\section{Limitation}

The results of the study should be interpreted keeping some limitations in mind. For instance, the small sample size of 18 clinical supervisors and 12 supervisees may make it difficult to generalize the findings. The study, however, has provided useful information about CS that can be further explored.

\section{Conclusion}

Clinical supervision helps promote practitioners' personal and professional development through fostering a supportive relationship and working alliance. Although, there are many benefits that can be reaped from effective clinical supervision, various personal and organisational challenges that inhibits effective supervision may also be encountered. The findings of this study could be useful to health institutions and providers such as hospitals and clinics involved in the training of students to achieve better clinical experience and skills acquisition.

\section{CONFlicts OF InTEREST Disclosure}

The authors declare that there is no conflict of interest.

\section{REFERENCES}

[1] Bifarin O, Stonehouse D. Clinical supervision: An important part of every nurse's practice. Br. J. Nurs. 2017; 26(6): 331335. PMid:28345984 https://doi.org/10.12968/bjon. 2017 .26 .6 .331

[2] Ning TJ, Costello J. Implementing clinical nursing supervision in Singapore hospitals. GSTF J Nurs Health Care Internet. 2017.

[3] Bohall G, Bautista MG. Clinical, Educational, and Administrative Supervision', in The Psychologist's Guide to Professional Development, Springer; 2017; 93-106. https ://doi .org/10.1007/97 8-3-319-63013-7_7

[4] Driscoll J, Stacey G, Harrison-Dening K, et al. Enhancing the quality of clinical supervision in nursing practice. Nurs. Stand. 2019. PMid:31468814 https://doi.org/10.7748/ns.2019.e11228

Published by Sciedu Press
[5] Baylis D. Why clinical supervision matters.... - Google Scholar. Pract. Nurse. 2014; 44(6): 29-30.

[6] Blishen M. "Why we need supervision". Nurs. N. Z. Wellingt. 1995; 22(2): 30-31.

[7] Martin P, Kumar S, Lizarondo L, et al. Enablers of and barriers to high quality clinical supervision among occupational therapists across Queensland in Australia: findings from a qualitative study. BMC Health Serv. Res. 2015; 15(1): 1-8. PMid:26404534 https://doi .org/10.1186/s12913-015-1085-8

[8] Koivu A, Hyrkäs K, Saarinen PI. Who attends clinical supervision? The uptake of clinical supervision by hospital nurses. J. Nurs. Manag. 2011; 19(1): 69-79. PMid:21223407 https://doi.org/10.111 $1 / j \cdot 1365-2834.2010 .01185 . x$

[9] Long CG, Harding S, Payne K, et al. Nursing and health-care assistant experience of supervision in a medium secure psychiatric 
service for women: Implications for service development. J. Psychiatr. Ment. Health Nurs. 2014; 21(2): 154-162. PMid:23551325 https://doi.org/10.1111/jpm.12066

[10] Watts R, Botti M, Hunter M. Nurses' perspectives on the care provided to cancer patients. Cancer Nurs. 2010; 33(2). PMid:20142746 https://doi.org/10.1097/NCC.0b013e3181b5575a

[11] Lyth GM. Clinical supervision: A concept analysis. J. Adv. Nurs. 2000; 31(3): 722-729. PMid:10718893 https://doi.org/10.1 $046 / j \cdot 1365-2648.2000 .01329 \cdot \mathrm{x}$

[12] Tomlinson J. Using clinical supervision to improve the quality and safety of patient care: A response to Berwick and Francis Career choice, professional education and development the Many Meanings of "Quality" in Healthcare: Interdisciplinary Perspectives. BMC Med Educ. 2015; 15(1). PMid:26062608 https://doi.org/10.1186/ s12909-015-0324-3

[13] Kilminster S, Cottrell D, Grant J, et al. AMEE Guide No. 27: Effective educational and clinical supervision. Med. Teach. 2007; 29(1) 2-19. PMid:17538823 https://doi .org/10.1080/0142159070 1210907

[14] Milne D. An empirical definition of clinical supervision. Br. J. Clin. Psychol. 2007; 46(4): 437-447. PMid:17535535 https ://doi .or $\mathrm{g} / 10.1348 / 014466507 \times 197415$

[15] Dawson M, Phillips B, Leggat S. Clinical supervision for allied health professionals: A systematic review. J. Allied Health. 2013; 42(2): 65-73.

[16] Galvin KT, Todres L. Research based empathic knowledge for nursing: A translational strategy for disseminating phenomenological research findings to provide evidence for caring practice. Int. J. Nurs. Stud. 2011; 48(4): 522-530. PMid:20863496 https: //doi.org/10.1016/j.ijnurstu.2010.08.009

[17] Creswell JW. Research design: Qualitative and mixed methods approaches. Lond. Thousand Oaks Sage Publ. 2009.

[18] Martin P, Copley J, Tyack Z. Twelve tips for effective clinical supervision based on a narrative literature review and expert opinion. Med. Teach. 2014; 36(3): 201-207. PMid:24256109 https: //doi.org/10.3109/0142159X.2013.852166

[19] King C, Edlington T, Williams B. The "ideal" clinical supervision environment in nursing and allied health. J. Multidiscip. Healthc. 2020; 13: 187-196. PMid:32110033 https://doi.org/10.2147/ JMDH . S239559

[20] Ekanoye A. Multicultural Issues in Supervision - Supervising International Counseling Students. Psychol. Behav. Sci. 2020; 9(2): 12 https://doi.org/10.11648/j.pbs.20200902.12

[21] O'Keeffe M, James M. Facilitated group supervision: Harnessing the power of peers. J. Paediatr. Child Health. 2014; 50(12): 944-948. PMid:24957327 https://doi .org/10.1111/jpc.12638
[22] Fernandez R, Sheppard-Law S, Curtis S, et al. Exploring the experiences of neophyte nurse mentors: A qualitative study. Nurse Educ. Pract. 2018; 29: 76-81. PMid:29197706 https ://doi .org/10.1 016/j.nepr.2017.11.011

[23] Snowdon DA, Millard G, Taylor NF. Effectiveness of clinical supervision of allied health professionals: A survey. J. Allied Health. 2016; 45(2): 113-121.

[24] O'Shea M, Kelly B. The lived experiences of newly qualified nurses on clinical placement during the first six months following registration in the Republic of Ireland. J. Clin. Nurs. 2012; 16(8): 1534-1542. PMid:17655542 https://doi.org/10.1111/j.1365-2702.20 06.01794. $\mathrm{x}$

[25] Cutcliffe JR, Sloan G, Bashaw M. A systematic review of clinical supervision evaluation studies in nursing. Int. J. Ment. Health Nurs. 2018; 27(5): 1344-1363. PMid:29446513 https://doi .org/10.1 111/inm. 12443

[26] Saab MM, et al. Peer group clinical supervision: Qualitative perspectives from nurse supervisees, managers, and supervisors. Collegian. 2020.

[27] Novoa-Gómez M, Córdoba-Salgado O, Rojas N, et al. A descriptive analysis of the interactions during clinical supervision. Front. Psychol. 2019; 10. PMid:30971991 https://doi.org/10.3389/fp syg. 2019.00669

[28] Ziba FA, Yakong VN, Ali Z. Clinical learning environment of nursing and midwifery students in Ghana. BMC Nurs. 2021; 20(1): 1-7. PMid:33413335 https://doi.org/10.1186/s12912-020-005 33-8

[29] Mathevula RF. Professional nurses' and student nurses' perceptions of clinical supervision in training hospitals of Limpopo Province: South Africa. PhD Thesis. 2019.

[30] McCarron RH, Eade J, Delmage E. The experience of clinical supervision for nurses and healthcare assistants in a secure adolescent service: Affecting service improvement. J. Psychiatr. Ment. Health Nurs. 2018; 25(3): 145-156. PMid:29266597 https ://doi.org/ $10.1111 / \mathrm{jpm} .12447$

[31] Neshuku H, Amukugo HJ. Experiences of registered and student nurses regarding the clinical supervision in medical and surgical wards: Develop an educational programme to support registered nurses. Int. J. Med. 2015; 3(2): 87-97. https ://doi .org/10.144 19/ijm.v3i2.5136

[32] Ducat WH, Kumar S. A systematic review of professional supervision experiences and effects for allied health practitioners working in nonmetropolitan health care settings. J. Multidiscip. Health. 2015; 8: 397 407. PMid:26347446 https : //doi .org/10.2147/JMDH . S84557

[33] Rahiem S, Habieb E, El-Shaer A. Impact of Clinical Supervision Program on Nursing Students' Achievement. IOSR J. Nurs. Health Sci. 2020 ; 9(1): 13-26. 